\title{
Identification of Car Body Steel by an optical on line System and a Kohonen's self-organizing Map
}

\author{
W. Kessler ${ }^{1}$, D. Ende', R. W. Kessler ${ }^{1}$, W. Rosenstiel ${ }^{2}$ \\ 'Institut fur Angewandte Forschung, FH Reutlingen \\ Alteburgst. 150, D-7410 Reutlingen, Germany \\ 2Institut für Technische Informatik, Universität Tübingen, \\ Sand 13, D-7400 Tübingen, Germany
}

The corrosion of materials is a result of the superposition of complex reactions which depend on surface thickness, its porosity and chemical composition. By means of optical spectroscopy, especially diffuse reflectance spectroscopy, the surface of low carbon steel can be analysed within milliseconds. From these results it is possible to predict its future corrosion resistance[1].

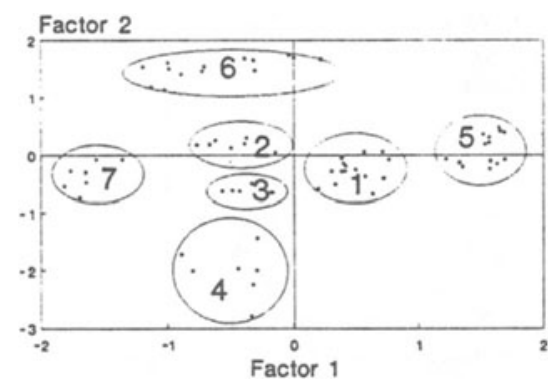

Figure 1: Classification of steel samples by the factor scores of the diffuse reflectance spectra

The pattern of the reflectance spectra in combination with a Kohonen's self-organizing map can be used to determine the corrosion behaviour of the steel sample as well as to typify the steel manufacturers. Steel samples from 7 different steel manufacturers (1 .. 7) in Europe were measured by the optical on line system. The reflectance spectra are analyzed by means of factor analysis and a Kohonen's self-organizing map.

Figure 1 shows the scores of the steel samples in the factor 1 versus factor 2 chart. The samples are classified by the factor scores. Factor 1 may be attributed to the chemical composition of the surface oxide (10 - $50 \mathrm{~nm}$ thickness) and factor 2 represents the porosity of the oxide. It is obvious that the 7 different manufacturers can be distinguished.

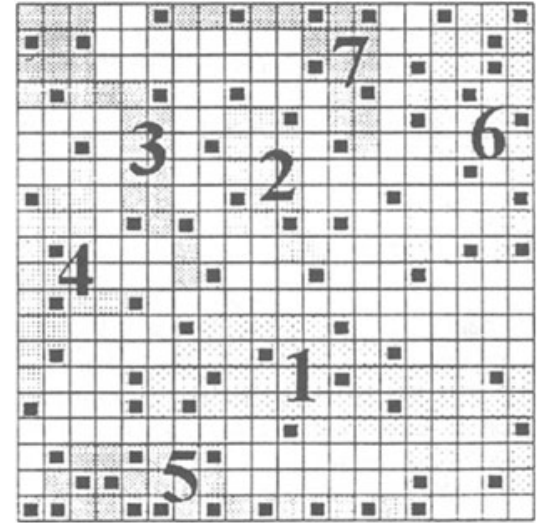

The Kohonen's self-organising map is as well used as a classifier. The map is trained with the same known input data, that were used for the factor analysis. An example of a classification with a Kohonen map is shown in figure. 2

The advantage of using the Kohonen map for classification compared to the factor analysis is a higher differentiation of the spectra especially of samples with high resemblance. The advantage of the factor analysis is the shorter computing time and the possibility of a scientific interpretation of the results.

Figure 2: Classification of car body steel samples by a Kohonen self-organizing map

\section{References}

[1] R. W. Kessler et al., Werkstoffe und Korrosion 40, 539 - 544 (1989) 